\title{
Perfil dos pacientes atendidos na Clínica Escola de Odontologia da Universidade de Gurupi nos últimos 2 (dois) anos
}

\section{Profile of patients seen at the Dental School of the University of Gurupi in the last 2 (two) years}

Caio Marques Mundim de Souza ; Matheus Bueno de Oliveira²; Vinicius Lopes Marinho $^{3}$

\section{RESUMO}

As clínicas escola odontológicas das Instituições de Ensino Superior (IES) vem buscando atender uma população que em sua grande maioria não possui condições sociais e econômicas de ter acesso a um tratamento dentário na iniciativa privada. Por essa razão, tais clínicas tem se tornado, essenciais nas buscas por melhores condições à saúde bucal da população. O estudo tem como objetivo detectar o perfil dos pacientes atendidos nas clínicas integradas I e II, na clínica escola de odontologia da Universidade de Gurupi, através de uma pesquisa documental caracterizada como quantitativo-descritiva, onde foram analisados 222 prontuários de usuários atendidos na Clínica Escola do curso de Odontologia da presente universidade no período de 2018 a 2019. No que diz respeito aos resultados, ficou evidente constatar que o perfil dos pacientes que utilizam os serviços disponíveis da clínica escola da Universidade de Gurupi são em sua maioria: mulheres, indivíduos acima de 50 anos, renda de até 1 (um) salário mínimo, com ensino fundamental incompleto e possuem diversas profissões. Além disso, as causas da busca para o tratamento odontológico são devido à dor de dente, assim como as condições clínicas pré-existentes mais predominantes foram a de hipertensão. E por fim, a especialidade mais procurada foi de dentística.

Palavras-chave: Pacientes. Perfil. Clínica Escola. Odontologia.

\section{ABSTRACT}

The dental school clinics of Higher Education Institutions (HEls) have sought to serve a population that, in the great majority, do not have the social and economic conditions to have access to dental treatment in the private sector. For this reason, such clinics have become essential in the search for better oral health conditions for the population. The study aims to detect the profile of patients seen in integrated clinics I and II in the dental school clinic of the University of Gurupi through a documentary research characterized as quantitative-descriptive where 222 medical records of users seen at the Clínica Escola of the Dentistry at the present university in the period from 2018 to 2019. Regarding the results, it was evident that the profile of patients who use the services available at the Gurupi University school clinic are mostly: women, individuals over 50 years old, income of up to 1 (one) minimum wage, with incomplete elementary education and have several professions. In addition, the causes for seeking dental treatment are due to toothache, as well as the most prevalent pre-existing clinical conditions of hypertension. And finally, the most sought after specialty outside dentistry.

Keywords: Patients. Profile. School Clinic. Dentistry.
1 Acadêmico do curso de Odontologia da Universidade UNIRG.

E-mail:

caiommundim123@gmail.com

Acadêmico do curso de Odontologia da Universidade UNIRG.

E-mail:

suehtambuenooliveira@outloo k.com

${ }^{3}$ Psicólogo, Doutorando em Ensino, Mestre em Ciências da Saúde-UFT, professor dos cursos de Psicologia e Odontologia da Universidade de Gurupi-TO.

E-mail:

viniciusmarinho@unirg.edu.br 


\section{INTRODUÇÃO}

No decorrer dos anos, a Odontologia vem se modernizando e sofrendo uma vasta mudança com alterações em relação ao seu método de atendimento à população mais carente, principalmente dentro de algumas classes sociais desprivilegiadas que dependem da assistência pública ou de entidades assistenciais, Organizações Não Governamentais (SILVA et., al.2015).

Apesar da reconhecida importância da saúde bucal, sabe-se que uma parcela considerável da população brasileira, assim como em outros países não tem acesso aos serviços de saúde, recorrendo a serviços públicos. No Brasil o desequilíbrio na distribuição de renda, somado à dificuldade dos serviços públicos de saúde de suprir a crescente demanda de pacientes em busca de atendimento odontológico, gera um grande aumento na procura pelas clínicas das IES (DA SILVA et al., 2015).

As Instituições de Ensino Superior (IES) em parceria com referidas entidades atuam como prestadoras de serviços para o atendimento a essas pessoas menos favorecidas ou de baixa renda, devendo atuar de acordo com o sistema de saúde vigente no país, disponibilizando-se como possibilidade de atendimento a vários usuários dos serviços de saúde.

Pelo fato de ter uma alta procura e não conseguir suprir toda a população, pacientes reivindicam as IES visando solucionar seus problemas e serem atendidos a contento, assim sendo houve um grande aumento na busca por essas instituições. Perante esta realidade, as clínicas odontológicas se esforçam no intuito de atender todas as demandas acadêmicas e dos usuários que as procuram, necessitando estar capacitadas para resolver os problemas dos usuários com condições sócio econômicas diferentes, além de diversas condições da saúde bucal (DA SILVA et al., 2015).

O fator sócio econômico está ligado à expectativa de vida e saúde. Algumas literaturas dizem que pode estar interligada também à saúde bucal. Evidências da correlação entre os indicadores de desenvolvimento social relacionados a renda, moradia e escolaridade, estão diretamente associados aos piores casos de saúde bucal, motivados pela falta de informação, ou até mesmo pela dificuldade da população em procurar instituições de atendimento. Portanto, compreender como cada indivíduo conhece a própria saúde é um importante passo para se esclarecer o padrão de procura dos serviços prestados nessa área (BRANDINI et al., 2008). 
A clínica escola de odontologia da Universidade de Gurupi possui pacientes de diferentes perfis e com demandas variadas de tratamento. Conhecer esses perfis de pacientes também é importante, pois permite visualizar como serão formados os CDs. Afinal, cada indivíduo possui uma história, hábitos e costumes diferentes.

Desta forma, formulou-se o seguinte problema: Qual perfil dos pacientes atendidos nas clínicas integradas I e II, na clínica escola de odontologia da Universidade de Gurupi?

Diante do exposto o presente estudo teve como objetivo investigar o perfil dos pacientes atendidos na clínica escola de odontologia da Universidade de Gurupi nos últimos dois anos.

\section{MATERIAIS E METODOS}

Tratou-se de uma pesquisa documental caracterizada como quantitativo-descritiva realizada junto a Clínica Escola do curso de Odontologia da Universidade de Gurupi.

Foram analisados 222 prontuários de usuários atendidos na Clínica Escola do curso de Odontologia da referida Universidade de Gurupi nos últimos 2 (dois) anos - 2018 e 2019. O recorte temporal foi determinado por conveniência, e por acreditar ter mais facilidade de acesso aos mesmos, por serem mais atuais.

Os critérios de inclusão dos prontuários foram: Prontuários de atendimentos realizados nos anos de 2018 e 2019; Prontuários com todos os campos fundamentais para realização da pesquisa devidamente preenchidos, e Prontuários legíveis. Já os critérios de exclusão foram aqueles que não se enquadrassem nos critérios pré-estabelecidos.

As variáveis extraídas dos prontuários para análise foram: gênero, faixa etária, nível de escolaridade, renda, profissão, motivo para procura do tratamento odontológico, condições clínicas pré-existentes e especialidade odontológica procurada.

Para a análise foi utilizado método quantitativo, onde foi efetuada análise descritiva dos dados (frequência e porcentagem) através do software SPSS - StatisticalPackage for the Social Sciences versão 20.0 para Windows, no qual os dados são apresentados em números absolutos e relativos e demonstrados em gráficos e tabelas.

\section{RESULTADOS E DISCUSSÃO}

No presente estudo foram analisados 222 prontuários de usuários atendidos na Clínica Escola do curso de Odontologia da Universidade de Gurupi, no período de 2018 a 
2019. Considerando a amostra analisada, os resultados obtidos por esse estudo, foram apresentados separadamente, no intuito de facilitar o entendimento dos mesmos.

Através da análise dos prontuários, obteve-se primeiramente, a classificação da amostra, segundo dados sóciodemográficos. Assim, apresenta-se abaixo a Tabela 1 que mostra os resultados desse item, a saber:

Tabela 1 - Variáveis Sociodemográficas

\begin{tabular}{|c|c|c|}
\hline $\begin{array}{l}\text { Variáveis sociodemográficas } \\
(\mathrm{n}=85)\end{array}$ & № de Usuários & Percentual \\
\hline \multicolumn{3}{|l|}{ Gênero } \\
\hline Masculino & 102 & $45,94 \%$ \\
\hline Feminino & 122 & $54,05 \%$ \\
\hline \multicolumn{3}{|l|}{ Faixa etária } \\
\hline Menores de 18 & 55 & $24,77 \%$ \\
\hline Entre 18 e 20 anos & 11 & $4,9 \%$ \\
\hline Entre 21 e 25 anos & 12 & $5,4 \%$ \\
\hline Entre 26 e 30 anos & 8 & $3,6 \%$ \\
\hline Entre 31 e 35 anos & 24 & $10,8 \%$ \\
\hline Entre 36 e 40 anos & 19 & $8,5 \%$ \\
\hline Entre 41 e 45 anos & 12 & $5,4 \%$ \\
\hline Entre 46 e 50 anos & 24 & $10,8 \%$ \\
\hline Acima de 50 anos & 58 & $26,12 \%$ \\
\hline \multicolumn{3}{|l|}{ Renda } \\
\hline Um salário mínimo & 101 & $45,4 \%$ \\
\hline Entre um e dois salários mínimos & 38 & $17,11 \%$ \\
\hline Entre dois e três salários mínimos & 15 & $6,7 \%$ \\
\hline Acima de tres salários mínimos & 2 & $0,9 \%$ \\
\hline Sem renda & 12 & $5,4 \%$ \\
\hline \multicolumn{3}{|l|}{ Nível de escolaridade } \\
\hline Não alfabetizado & 8 & $3,6 \%$ \\
\hline Ensino Fundamental incompleto & 98 & $44,14 \%$ \\
\hline Ensino Fundamental completo & 12 & $5,4 \%$ \\
\hline Ensino Médio incompleto & 59 & $26,5 \%$ \\
\hline Ensino Medio completo & 18 & $8,1 \%$ \\
\hline Ensino superior incompleto & 7 & $3,5 \%$ \\
\hline Ensino superioR completo & 14 & $6,3 \%$ \\
\hline Pós-graduação & 1 & $0,45 \%$ \\
\hline Não constava nível de escolaridade & 5 & $2,25 \%$ \\
\hline \multicolumn{3}{|l|}{ Profissão } \\
\hline Do lar & 15 & $6,7 \%$ \\
\hline Diarista & 18 & $8,1 \%$ \\
\hline Autonômos & 6 & $2,7 \%$ \\
\hline Desempregados & 23 & $10,3 \%$ \\
\hline Estudantes & 57 & $25,6 \%$ \\
\hline
\end{tabular}




\begin{tabular}{llr}
\hline Aposentados & 25 & $11,2 \%$ \\
\hline Outros & 78 & $35,13 \%$
\end{tabular}

Fonte: Dados primários (2020)

Em relação aos dados da tabela 1, que tratam do perfil dos pacientes atendidos pode-se afirmar que os mesmos são importantes, porque ajudam a entender o perfil das pessoas atendidas nessas unidades. Neste sentindo, Rossato; Bellini (2014) afirmam que o conhecimento sobre os dados sociais, demográficos e econômicos da população facilitaria o entendimento dos principais tipos de procedimentos clínicos realizados na Universidade, assim como o planejamento logístico e de recursos materiais e humanos para oferecer excelência na prestação de serviços à comunidade local.

No que se refere ao perfil sociodemográfico dos pacientes, verificou-se que em relação ao gênero, as mulheres são as que mais procuram o atendimento na Clínica Escola de Odontologia da Universidade de Gurupi, com $54,05 \%$. Comparando esse fato com outros estudos coletados, todos mostraram o mesmo resultado.

No estudo de Pombo et al. (2019) o gênero feminino foi o que mais procurou atendimento $(64,7 \%)$. No trabalho desenvolvido por Nakamura et al. (2010) foram avaliados 1.019 prontuários de pacientes atendidos no ano de 2007 , dos quais $59 \%$ dos pacientes eram do gênero feminino e $41 \%$ do gênero masculino.

A mesma alta porcentagem no gênero feminino também foi encontrado no estudo de Rossato; Bellini (2014) ao qual a maior parte dos indivíduos que recorrem aos serviços de saúde em uma Instituição de Ensino Superior - IES, é formada pelo gênero feminino $(65,66 \%)$. Em resultado idêntico ao último, no estudo de Silva et al. (2019) os achados mostraram que os usuários atendidos em clínica-escola são, predominantemente, do sexo feminino (65,5\%). Por fim, no estudo de Melo et al. (2014) houve prevalência de pacientes do gênero feminino $(60 \%)$ atendidos pelo serviço de triagem da Clínica de Estomatologia no curso de Odontologia da Universidade Vale do Rio Verde (UninCor).

Sanchez e Drumond (2011) consideram que a explicativa por essa unanimidade é oriunda de vários fatores. Primeiramente, é justificado pelo fato de haver maior prevalência de mulheres na população brasileira, e por provavelmente se preocuparem mais com a saúde e a estética que os homens.

Esse resultado também pode estar associado à "questão cultural ou social, em que a mulher normalmente é responsável por acompanhar o filho e os idosos ao médico, além 
de realizar o pré-natal, tornando-a naturalmente mais disposta a frequentar os serviços de saúde" (ROSSATO; BELLINI, 2014, p. 09).

No caso dos homens, Silva et al. (2019) afirmam que o medo da descoberta de uma doença grave e a vergonha da exposição do corpo perante o profissional de saúde, contribuem para a pouca procura dos homens ao serviço de assistência.

No que diz respeito à faixa etária, a maior evidencia foi a dos pacientes com mais de 50 anos, totalizando $26,12 \%$ dos participantes, seguida dos pacientes menores de 18 anos, que corresponderam a um total de $24,77 \%$ da amostra estudada. Os achados deste estudo em relação a essa variável foram na contramão apresentados por outros estudos, onde o público era formado em sua grande maioria por adultos (entre 30 e 40 anos).

A título de exemplo, pode ser citado o estudo divulgado por Nakamura et al. (2010) onde foi verificado que pacientes adultos jovens, com idade entre 20 e 29 anos (31\%), e adultos de 30 a 39 anos (22\%) foram os que mais procuraram o serviço odontológico. Corroborando com esse resultado, na pesquisa de Rossato; Bellini (2014) a faixa etária dos entrevistados do estudo encontrava-se na faixa etária de 41 a 50 anos.

Já no estudo de Pombo et al. (2019, p. 03) que também teve essa similaridade, ele explica que esse resultado se deve pelo fato de que "os idosos costumam ter mais dificuldade de deslocamento até a localidade onde acontecem os atendimentos". De todo modo, na pesquisa aqui analisada, o público alvo ainda é o que possui idade maior de 50 anos, o que pode ser explicado pela atenção dispensada desses indivíduos em melhor buscar ajuda profissional no cuidado dentário.

Os dados colhidos relacionados à renda, a maioria dos pacientes relataram receber um salário mínimo (101 pacientes, com a porcentagem de 45,4\% do total), sendo acima de três salários mínimos ( 2 pacientes, 0,9\% do total), os que não apresentam renda mensal (sem renda) foram 12 pacientes totalizando 5,4\%. Há ainda os que revelaram ter ganhos mensais entre um e dois salários mínimos $(17,11 \%)$ e entre dois e três salários mínimos $(6,7 \%)$.

Sobre o dado socioeconômico, esse estudo apontou que a grande parcela dos pacientes possui renda de até 1 (um) salário mínimo (45,4\%). Isso vai de encontro a alguns estudos com o mesmo propósito conforme podem ser evidenciados. Em estudo realizado por Gabardo et al. (2015) investigando a associação entre as variáveis sociodemográficas, socioeconômicas, psicossociais e comportamentais e a saúde bucal, foi possível concluir 
que a autopercepção individual de saúde bucal mais desfavorável foi relatada por mulheres, por pessoas com idade mais avançada e por indivíduos com baixa renda familiar.

Esse resultado pode ser explicado, em parte, devido ao fato de que no imaginário coletivo, o serviço prestado pelos Cursos de Odontologia seria destinado somente para indivíduos sem condições financeiras, para receber atendimento odontológico particular (ROSSATO; BELLINI, 2014). Porém, essa não é a realidade do Curso de Odontologia da Universidade de Gurupi, uma vez que o atendimento é destinado a toda população, independentemente da condição social ou financeira.

O nível de escolaridade mais descrito foi o ensino fundamental incompleto (98 pacientes, com 44,14\% do total); já o que não constava nível de escolaridade foi o menos descrito (5 pacientes, com 2,25\% do total). Além destes, cita-se ainda os não alfabetizados (3,6\%); Ensino Fundamental completo (5,4\%); Ensino Médio incompleto (26,5\%); o Ensino Médio completo (8,1\%); o Ensino Superior incompleto (3,5\%); o Ensino Superior completo (6,3\%) e Pós-Graduação (0,45\%).

No que tange à escolaridade, nota-se que no estudo em análise a porcentagem maior $(44,14 \%)$ foi daqueles pacientes que tinham o ensino fundamental incompleto. Esse dado pode estar relacionado ao fato de que esses indivíduos possuem baixa renda, uma vez que é possível assentir que quanto menor é o poder aquisitivo, menor também será o nível de escolaridade.

No estudo de Rossato; Bellini (2014) o grau de instrução dos pacientes estudados, a maioria dos entrevistados possui até a 4. a série $(34,85 \%)$ e apenas $3,54 \%$ dos mesmos possuem nível superior. Esses autores interpretam esse resultado afirmando que "os indivíduos com maior grau de escolaridade, geralmente, possuem melhor condição financeira e não se submetem a procedimentos realizados por alunos em fase de aprendizagem" (ROSSATO; BELLINI, 2014, p. 10), o que também é corroborado por essa pesquisa.

A profissão mais evidente foi a de estudante (57 pacientes, $25,6 \%$ do total). Por fim, os pacientes que apresentaram profissões diversificadas e menos evidente na pesquisa foram apresentados como outros (78 pacientes, 35,13\% do total).

No que se refere à profissão, o presente estudo apontou que não há uma profissão única perante os pacientes pesquisados, ou seja, o grupo majoritário $(35,13 \%)$ é descrito por outras profissões diversas das disponíveis. Dentre as que se encontravam como opções na pesquisa, houve um público predominantemente formado por estudantes $(25,64 \%)$. 
Esse resultado aponta para o fato de que muitos estudantes da área da saúde, incluindo aí a de Odontologia, acabam se tornando também pacientes nas clínicas-escola.

Em seguida buscou-se identificar quais as motivações que levam os pacientes a procurarem atendimento dentário na Clínica Escola de Odontologia da Universidade de Gurupi. Os resultados encontrados foram:

Gráfico 1- Motivo da procura para tratamento odontológico

$\begin{array}{ll}\text { - Dor de dente } & \text { Dente cariado } \\ \text { Dente quebrado ou restaurado } & \text { - Outros } \\ \square \text { Fazer tratamento endodontico } & \square \text { Fazer extraçao } \\ \square \text { Fazer limpeza } & \text { Fazer uma protese nova }\end{array}$

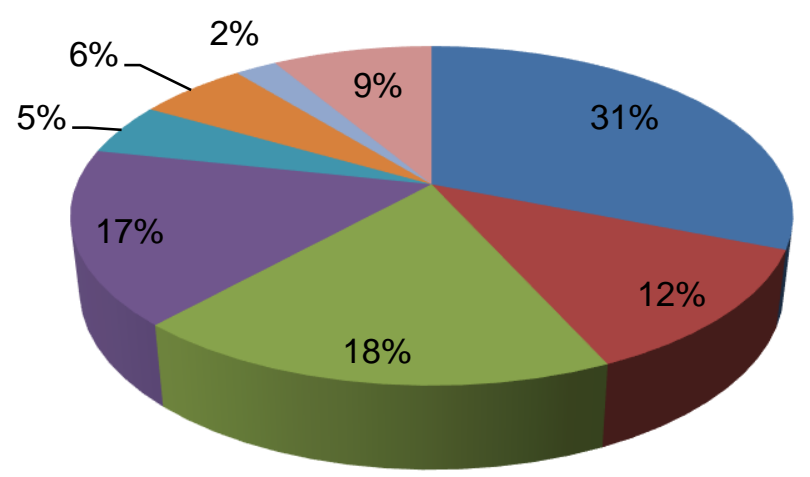

Fonte: Dados primários (2020)

Dentre os prontuários, os motivos de tratamento mais procurados foi dor de dente com $31 \%$ dos pacientes, sendo o menos procurado o de fazer limpeza (2\%). Os pacientes que apresentaram tipos variáveis de procura classificados com outros foram $(17 \%)$ do total de pacientes. Além destes, tem-se ainda o dentre quebrado ou restaurado (18\%); fazer tratamento endodôntico (5\%); dente cariado (12\%); fazer extração (6\%) e por fim, fazer uma prótese nova (9\%).

Prosseguindo, a motivação principal para a procura de tratamento odontológico na clínica escola de Odontologia da presente Universidade é em razão da dor de dente (31\%). Esse resultado é semelhante ao encontrado pelos estudos agrupados.

No estudo de Rossato; Bellini (2014) foi constatado que a maior parte dos pacientes entrevistados $(35,86 \%)$ relatou ter procurado atendimento na Clínica de Odontologia na UNIARA pelo motivo de dor de dente. Em outro dado semelhante, na pesquisa de Silva et 
al. (2019) apontou que a "dor de origem dentária" foi o principal motivo para a busca pelo serviço para $35,1 \%$ dos pacientes pesquisados.

Melo (2014) explica que esse resultado mostra que a população em geral busca um tratamento odontológico apenas em situações de emergência, ou seja, quando há uma evidência de dor. Ferreira et al. (2012) acrescenta ainda que a dor de dente é causada por vários fatores, mas destaca que se deve sobretudo, à baixa frequência de escovação diária, ao alto intervalo entre as consultas odontológicas, à ansiedade odontológica, ao consumo de alimentos cariogênicos, à alta incidência de cárie e à presença de lesão cariosa não tratada.

A próxima questão pesquisada diz respeito às condições clínicas pré-existentes, que de modo geral é toda condição onde o indivíduo já possui e sabe disso antes da contratação do serviço. Ou seja, trata-se de qualquer doença que tenha sido descoberta antes do atendimento na clínica escola (GALVAN, 2019).

Gráfico 2 - Condições clínicas pré-existentes
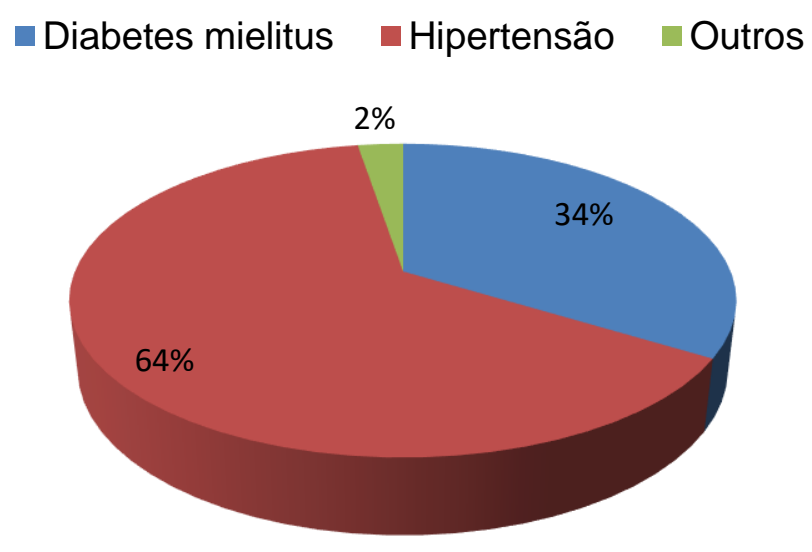

Fonte: Dados primários (2020)

Quando se analisa os números de condições clínicas pré-existentes, foi observado que a maioria dos pacientes apresentava hipertensão (64\%). Já 34\% dos pacientes apresentavam diabetes mellitus, $2 \%$ dos pacientes tinham outros tipos de condições clínicas pré-existentes, como osteoporose, febre reumática, hepatite, artrose, dentre outros.

Neste Gráfico encontramos as informações relacionadas às condições clínicas préexistentes, assim sendo a maioria dos pacientes apresentava hipertensão (64\%). Esse 
resultado é compreensível, uma vez que a hipertensão é uma das doenças que mais acometem os indivíduos (GUTIERRE, 2017).

Também denominada de pressão alta, a hipertensão é quando a pressão que o sangue exerce nas paredes das artérias para se movimentar é muito forte, ficando acima dos valores considerados normais. Além disso, essa doença implica no endurecimento das paredes vasculares, o que dificulta a passagem do fluxo sanguíneo (GUTIERRE, 2017).

Ademais, a última questão analisada foi no que tange à especialidade. Nessa questão, se buscou identificar qual área dentro da Odontologia que mais teve procura pelos pacientes, ou seja, qual especialidade odontológica mais se realizou durante o período coletado.

Os resultados mostrados a seguir, trazem um dado interessante: há uma similaridade de porcentagens entre as áreas, o que mostra que a procura na Clínica Escola de Odontologia da Universidade de Gurupi é diversa e equilibrada, tendo sido realizadas cirurgias, periodontia, dentística, odontopediatria, entre outros.

\section{Gráfico 3 - Especialidade}

\begin{tabular}{|c|c|c|}
\hline gia & - Periodontia & Endodontia \\
\hline
\end{tabular}

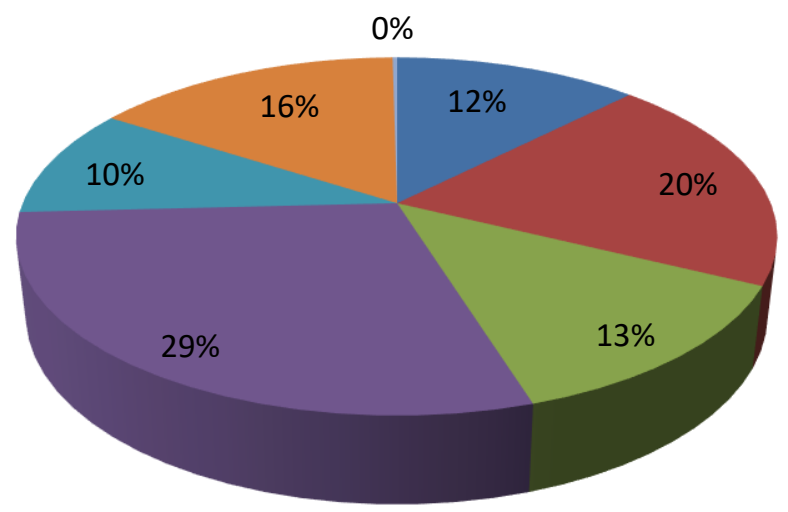

Fonte: Dados primários (2020)

Como já mencionado anteriormente, há um equilíbrio no resultado dessa questão. Conforme ilustra o Gráfico 3 a especialidade mais procurada pelos pacientes foram dentística (29\%), seguida pela periodontia (20\%), prótese (16\%), endodontia (13\%), cirurgia 
(12\%) e odontopediatria (10\%). A categoria outros $(0 \%)$ se refere a outras especialidades de dor orofacial.

Conceitualmente, a dentística ou odontologia estética é o ramo da odontologia que atua na área da cosmética e restauração dental. Entre outros serviços, os profissionais desta especialidade tratam de clareamentos dos dentes, uso de resinas diretas, facetas, lentes de contato dental, e restaurações estéticas (SILVA; SILVA; BARBOSA, 2017).

O dado mostrado por esse estudo foi semelhante ao apresentado por Pombo et al. (2019) que em seu trabalho, em relação às demandas por atendimento referente à especialidade, a dentística predominou com $89,5 \%$. Isso é explicado pelo fato de que a população em geral se preocupa mais com a estética dentária do que outros assuntos relacionados à área. O sorriso é considerado a porta de entrada da estética das pessoas, portanto, ter um dente branco e limpo, se torna essencial. Por se tratar de uma clínica escola que trata de quase todo tipo de tratamento dentário, os pacientes no geral acabam buscando a melhoria estética de seus dentes, como bem mostrou o resultado dessa pesquisa.

Diante de todo o exposto, fica evidente constatar que o perfil dos pacientes que utilizam os serviços disponíveis da clínica escola da Universidade Gurupi são em sua maioria: mulheres, indivíduos acima de 50 anos, renda de até 1 (um) salário mínimo, com ensino fundamental incompleto e possuem diversas profissões. Além disso, as causas para a busca para o tratamento odontológico são devido à dor de dente, assim como as condições clínicas pré-existentes mais predominantes foram a de hipertensão. E por fim, a especialidade mais procurada foi de dentística.

\section{CONSIDERAÇOES FINAIS}

Em relação ao perfil dos pacientes atendidos na Clínica Escola de Odontologia da Universidade de Gurupi, verificou- se que a maioria tratava-se do gênero feminino; faixa etária acima de 50 anos; renda de até 1 (um) salário mínimo; possuem o ensino fundamental incompleto, possuem diversos cargos de trabalho, sendo o de estudante o que apresentou maior porcentagem dentre os disponíveis na pesquisa; procuraram atendimento odontológico na Instituição estudada pelo motivo de dor de dente; a condição pré-existente mais encontrada foi a hipertensão; a especialidade mais solicitada é a de dentistica.

Diante disso, os resultados encontrados são relevantes ao despertar a atenção para o conhecimento do perfil clínico e epidemiológico dos pacientes que buscam na clínica 
escola de Odontologia da Universidade de Gurupi o tratamento para os seus dentes, sendo ainda de grande importância para a presente instituição de ensino superior pesquisado.

Dessa forma, o presente estudo forneceu conhecimento sobre o perfil socioeconômico dos pacientes atendidos na Clínica Escola de Odontologia da Universidade de Gurupi, o qual poderá ser utilizado para elevar a qualidade de planejamento dos atendimentos clínicos oferecidos à população que anualmente recorre ao Curso de Odontologia da presente universidade.

\section{REFERENCIAS}

FERREIRA, LL. et al. Odontalgia associada a variáveis socioeconômicas, psicossociais e saúde bucal. Revista Dor, 2012:13(40:343-9). Disponível em: <http://repositorio.ufpa.br/jspui/handle/2011/7750>. Acesso em: 14 dez. 2020.

GABARDO, MCL. et al. Social, economic, and behavioral variables associated with oral health-related quality of life among Brazilian adults. Ciência\&saúde coletiva, 2015. 20(5): 1531-1540. Disponível em: <http://www.scielo.br/scielo.php?pid=S141381232015000501531\&script=sci_arttext\&tlng= es>. Acesso em: 14 dez. 2020.

GALVAN, Jessica. Pré-natal odontológico de gestantes de alto risco: análise sob o prisma de diferentes fatores. Dissertação apresentada para a obtenção do título de Mestre em Ciências da Saúde na Universidade Estadual de Ponta Grossa, Área de Assistência Interdisciplinar em Saúde, Linha de pesquisa Assistência Integral à Saúde e Qualidade de Vida. Ponta Grossa, 2019. Disponível em: <https://tede2.uepg.br/jspui/bitstream/prefix/2809/1/Jessica\%20Galvan.pdf>. Acesso em: 14 dez. 2020.

GUTIERRE, Dental. O Paciente Hipertenso e a Odontologia. 2017. Disponível em: $<$ http://blog.dentalgutierre.com.br/o-paciente-hipertenso-e-a-odontologia/>. Acesso em: 14 dez. 2020.

MELO, Júlia Campos; et al. Perfil dos pacientes atendidos na clínica odontológica da UNINCOR. Revista da Universidade Vale do Rio Verde, Três Corações, v. 12, n. 1, p. 614620, jan./jul. 2014.

NAKAMURA, Clayton Cézar; et al. Perfil dos pacientes atendidos na clínica odontológica da Faculdade São Lucas, Porto Velho - RO. Saber Científico Odontológico, Porto Velho, 1 (1): 42 - 52, jul./dez.,2010.

POMBO, Stephanie Quintans da Rocha et al. Perfil dos Pacientes Atendidos no Curso de Odontologia do Sertão de Pernambuco - Perfil dos Pacientes Atendidos no Sertão.Rev. Cir. Traumatol. Buco-Maxilo-Fac., Camaragibe v.19, n.2, p. 6-12, abr./jun. 2019. 
ROSSATO, Emersom Mocheti; BELLINI, Afonso. Levantamento do perfil social, demográfico e econômico de pacientes atendidos na clínica de odontologia do centro universitário de Araraquara - UNIARA. Revista UNIARA, v.17, n.1, julho 2014.

SANCHEZ, H.F; DRUMOND, M.M. Atendimento de urgências em uma Faculdade de Odontologia de Minas Gerais: perfil do paciente e resolutividade. Rev Gaúcha Odontol. 2011;59(1):79-86.

SILVA, Bianca dos Santos; et al. Perfil epidemiológico e saúde bucal de pacientes atendidos em uma clínica integrada de odontologia. Revista da Revista da Universidade Vale do Rio Verde. v. 17 n.1 jan./jul. 2019.

SILVA, Juliana Cândido; SILVA, Diego Romário; BARBOSA, Danielle Nascimento. Estabilidade de cor das resinas compostas: um desafio para a dentística restauradora. Arch Health Invest (2017) 6(10):451-457. 